\title{
THE FOURIER TRANSFORM OF VECTOR-VALUED FUNCTIONS
}

\author{
by SUSUMU OKADA
}

(Received 11 September, 1984)

For each natural number $n$, let $u_{n}(x)=(1-\cos n x) / \pi n x^{2}(x \in \mathbb{R})$. It is well-known that a bounded continuous function $f$ on the real line $\mathbb{R}$ is the Fourier transform of an integrable function on $\mathbb{B}$ if and only if the functions $\Phi_{n}(f)(n=1,2, \ldots)$, defined by

$$
\Phi_{n}(f)(x)=\frac{1}{2 \pi} \int_{-\infty}^{\infty} e^{i x t} f(t) \hat{u}_{n}(t) d t, \quad(x \in \mathbb{R}),
$$

form a Cauchy sequence in the space $\mathscr{L}_{1}(\mathbb{R})$ (cf. [2]). Such a characterization, which can be extended to functions defined on a locally compact Abelian group more general than $\mathbb{R}$, is based on the fact that the space $\mathscr{L}_{1}(\mathbb{R})$ is complete with respect to convergence in mean.

However, as noted in [6], this criterion cannot be extended to characterize the Fourier transforms of vector-valued, Pettis integrable functions. This is due to the fact that the space of Pettis integrable functions is not complete with respect to convergence in mean (cf. [8]).

The integral introduced in [7] does not suffer from this defect. It leads to a space of integrable functions which is complete with respect to convergence in mean. Accordingly, the Fourier transforms of such vector-valued, integrable functions can be characterized by a criterion analogous to that which characterizes the Fourier transforms of scalar-valued integrable function. The aim of this note is to present such a criterion.

1. The Archimedes integral. Let $\lambda$ be a choice of Haar measure in a locally compact Abelian group $G$. Let $X$ be a complex Banach space and $X^{\prime}$ its dual space.

Let $Y$ be a locally convex Hausdorff space into which the space $X$ is continuously embedded. A function $F: G \rightarrow Y$ is called Archimedes integrable with respect to $\lambda$ in the space $X$, briefly $(X, \lambda)$-integrable, if there exists a function $H: G \rightarrow Y$, vectors $c_{i} \in X$ and Borel subsets $E_{i}$ of $G,(i=1,2, \ldots)$, such that

(i) the function $F$ is $\lambda$-almost everywhere equal to $H$;

(ii) the sequence $\left\{c_{i} \lambda\left(E_{i}\right)\right\}_{i \in \mathbb{N}}$ is unconditionally summable in the space $X$; and

(iii) if $y^{\prime} \in Y^{\prime}$, then

$$
\left\langle y^{\prime}, H(g)\right\rangle=\sum_{i=1}^{\infty}\left\langle y^{\prime}, c_{i}\right\rangle \chi_{E_{i}}(g),
$$

for every $g \in G$ for which

$$
\sum_{i=1}^{\infty}\left|\left\langle y^{\prime}, c_{i}\right\rangle\right| \chi_{E_{i}}(g)<\infty
$$

Glasgow Math. J. 26 (1985) 181-186. 
The $X$-valued set function $F \lambda$ defined by

$$
(F \lambda)(E)=\sum_{i=1}^{\infty} c_{i} \lambda\left(E_{i} \cap E\right),
$$

for every Borel set $E$, is called the indefinite integral of the function $F$ with respect to $\lambda$. Since $Y^{\prime}$ separates points of $X$, the indefinite integral $F \lambda$ is well-defined (cf. [7, Lemma 1, Note 11]). By the Vitali-Hahn-Saks theorem, it is $\sigma$-additive. We shall also use the classical notation

for each Borel set $E$.

$$
(F \lambda)(E)=\int_{E} F d \lambda
$$

The vector space of all $Y$-valued, $(X, \lambda)$-integrable functions on $G$ is denoted by $\mathscr{L}(\lambda ; X, Y)$. Define the seminorm $\|\cdot\|$ on the space $\mathscr{L}(\lambda ; X, Y)$ by

$$
\|F\|=\sup \left\{\left|\left\langle x^{\prime}, F \lambda\right\rangle\right|(G):\left|x^{\prime}\right| \leq 1, x^{\prime} \in X^{\prime}\right\},
$$

for every $F \in \mathscr{L}(\lambda ; X, Y)$, where $\left|\left\langle x^{\prime}, F \lambda\right\rangle\right|$ denotes the total variation of the complex measure $\left\langle x^{\prime}, F \lambda\right\rangle$. The topology on $\mathscr{L}(\lambda ; X, Y)$ given by this seminorm is called the topology of convergence in mean. It is clear that the space of $X$-valued, $(X, \lambda)$-integrable Borel simple functions on $G$ is dense in the space $\mathscr{L}(\lambda ; X, Y)$ (cf. [7, Proposition 2]).

There always exists a locally convex Hausdorff space $Y$, into which $X$ is continuously embedded, such that the space $\mathscr{L}(\lambda, X, Y)$ is complete with respect to convergence in mean. For example, if $\Theta$ is a total subset of the dual space $X^{\prime}$, then $X$ is continuously embedded into the product space $\mathbb{C}^{\Theta}$ and we have the following result.

THEOREM 1.1 ([7, Theorem 5]). The space $\mathscr{L}\left(\lambda ; X, \mathbb{C}^{\Theta}\right)$ is complete with respect to convergence in mean.

The relationship between the Pettis integral and the Archimedes integral is given by the following result.

Proposition 1.2 ([7, Proposition 14]). A function $F: G \rightarrow X$ is $(X, \lambda)$-integrable if and only if it is strongly measurable and Pettis integrable with respect to $\lambda$. Moreover, the indefinite integral $F \lambda$ of each function $F \in \mathscr{L}(\lambda ; X, X)$ is equal to the indefinite Pettis integral of $F$.

2. The Fourier transform. Let $G$ be a locally compact Abelian group and $\Gamma$ its dual group. Let $\lambda$ be a fixed Haar measure in the group $G$. Let $\nu$ be the Haar measure in $\Gamma$ which is so normalized that the inversion formula is valid (cf. [9, Theorem I.5.1]).

Throughout this section, $X$ is a Banach space and $Y$ is a locally convex Hausdorff space into which $X$ is continuously embedded.

For each function $F \in \mathscr{L}(\lambda ; X, Y)$, let

$$
\hat{F}(\gamma)=\int_{G} F(g)(-g, \gamma) d g \quad(\gamma \in \Gamma)
$$


The $X$-valued function $\hat{F}$ so defined is called the Fourier transform of the function $F$. It exists by [7, Corollary 4$]$.

According to [4, Theorem 33.12], the Banach algebra $\mathscr{L}_{1}(G)$ of all complex-valued, $\lambda$-integrable functions on $G$ admits an approximate unit $\left\{u_{\alpha}\right\}_{\alpha \in A}$ such that

(i) for every $\alpha \in A$, the function $u_{\alpha}$ is non-negative and $\left(u_{\alpha} \lambda\right)(G)=1$;

(ii) for every $\alpha \in A$, the Fourier transform $\hat{u}_{\alpha}$ of $u_{\alpha}$ is a continuous function on $\Gamma$ with compact support;

(iii) for every $\alpha \in A$, the function $u_{\alpha}$ is equal to the inverse Fourier transform of $\hat{u}_{\alpha}$;

(iv) $\lim _{\alpha \in A} \hat{u}_{\alpha}=1$ on $\Gamma$, the convergence being uniform on all compact subsets of $\Gamma$.

Let $\alpha \in A$. Suppose that $f: \Gamma \rightarrow X$ is a weakly continuous map. Then, for every $g \in G$, the $X$-valued function $\gamma \mapsto f(\gamma) \hat{u}_{\alpha}(\gamma)(\mathrm{g}, \gamma)$, where $\gamma \in \Gamma$, is Pettis $\nu$-integrable since it has weakly compact range (cf. [5, Lemma 4$]$ or $[3$, p. 88]). Let

$$
\Phi_{\alpha}(f)(g)=\int_{T} f(\gamma) \hat{u}_{\alpha}(\gamma)(g, \gamma) d \gamma \quad(g \in G),
$$

where the right hand side of (1) is the Pettis integral over $\Gamma$.

THEOREM 2.1. Let $f: \Gamma \rightarrow X$ be the Fourier transform of an element of $\mathscr{L}(\lambda ; X, Y)$. Then $f$ is a bounded continuous function vanishing at infinity, and the $X$-valued functions $\Phi_{\alpha}(f)$, where $\alpha \in A$, given by (1) are $(X, \lambda)$-integrable and form a Cauchy net in the space $\mathscr{L}(\lambda ; X, X)$ with respect to convergence in mean.

Proof. Take a function $F \in \mathscr{L}(\lambda ; X, Y)$ such that $\hat{F}=f$. Then there exist $(X, \lambda)$ integrable, Borel simple functions $H_{n}: G \rightarrow X(n=1,2, \ldots)$, which are convergent in mean to the function $F$. It follows that, for every natural number $n$,

$$
\left|f(\gamma)-\hat{H}_{n}(\gamma)\right| \leq\left\|F-H_{n}\right\| \quad(\gamma \in \Gamma) .
$$

For every natural number $n$, the function $\hat{H}_{n}$ is continuous and vanishes at infinity since $H_{n}$ is a simple function (cf. [9, Theorem I.2.4]). It follows from (2) that the function $f$ is also continuous and vanishes at infinity.

Let $\alpha \in A$. By [6, Lemma 7], the function $\Phi_{\alpha}(f)$ is Pettis $\lambda$-integrable. We claim that $\Phi_{\alpha}(f)$ has relatively compact range in the space $X$. Indeed, the bounded function $f \hat{u}_{\alpha}$ with compact support is Bochner $\nu$-integrable and its indefinite Bochner integral has relatively compact range $R$ in $X$ (cf. [3, Theorem VIII.1.5]). Consequently, the range of $\Phi_{\alpha}(f)$ is included in the compact set $4 \mathrm{bco} R$, where bco $R$ denotes the closed balanced convex hull of the set $R$.

Since the function $\Phi_{\alpha}(f)$ is scalarly $\lambda$-integrable and has separable range, it vanishes $\lambda$-almost everywhere outside some Borel set of $\sigma$-finite measure. By the Pettis measurability theorem, the function $\Phi_{\alpha}(f)$ is strongly $\lambda$-measurable. It follows from Proposition 1.2 that the function $\Phi_{\alpha}(f)$ is $(X, \lambda)$-integrable.

For every $\alpha \in A$ and every natural number $n$,

$$
\left\|\Phi_{\alpha}(f)-\Phi_{\alpha}\left(\hat{H}_{n}\right)\right\| \leq\left\|F-H_{n}\right\| .
$$


In fact, the strongly $\lambda$-measurable functions $\Phi_{\alpha}(f)$ and $\Phi_{\alpha}\left(\hat{H}_{n}\right)$ both vanish $\lambda$-almost everywhere outside a Borel set $E$ of $\sigma$-finite measure. Let $\mu=\left(F-H_{n}\right) \lambda$. Since $\hat{u}_{\alpha}$ has compact support, the Fubini theorem ensures that, for every $x^{\prime} \in X^{\prime}$,

$$
\begin{aligned}
\int_{G}\left|\left\langle x^{\prime}, \Phi_{\alpha}(f)-\Phi_{\alpha}\left(\hat{H}_{n}\right)\right\rangle\right| d \lambda & =\int_{E}\left|\int_{G} d\left\langle x^{\prime}, \mu\right\rangle(h) \int_{T} \hat{u}_{\alpha}(\gamma)(g-h, \gamma) d \gamma\right| d g \\
& =\int_{E}\left|\int_{G} u_{\alpha}(g-h) d\left\langle x^{\prime}, \mu\right\rangle(h)\right| d g \\
& \leq \int_{G} d\left|\left\langle x^{\prime}, \mu\right\rangle\right|(h) \int_{G} u_{\alpha}(g-h) d g \leq\left|x^{\prime}\right|\left\|F-H_{n}\right\| .
\end{aligned}
$$

Thus, the inequality (3) is valid.

Let $n$ be a natural number. Since $H_{n}$ is a simple function, it follows from [10, Theorem 2(a) $]$ that the net $\left\{\Phi_{\alpha}\left(\hat{H}_{n}\right)\right\}_{\alpha \in \mathrm{A}}$ is Cauchy in the space $\mathscr{L}(\lambda ; X, X)$. By (3), the net $\left\{\Phi_{\alpha}(f)\right\}_{\alpha \in A}$ is also Cauchy in the space $\mathscr{L}(\lambda ; X, X)$.

The statement in Theorem 2.1 that the Fourier transform of an Archimedes integrable function vanishes at infinity is a vector version of the classical Riemann-Lebesgue lemma.

A sufficient condition for an $X$-valued function on $\Gamma$ to be the Fourier transform of an Archimedes integrable function on $G$ is given by the following result.

THEOREM 2.2. Let $\Theta$ be a total subset of the dual space $X^{\prime}$ and $Y=\mathbb{C}^{\Theta}$. Let $f: \Gamma \rightarrow X$ be a bounded weakly continuous function such that, for every $\alpha \in A$, the function $\Phi_{\alpha}(f): G \rightarrow X$, given by (1), is $(X, \lambda)$-integrable and the net $\left\{\Phi_{\alpha}(f)\right\}_{\alpha \in \mathbb{A}}$ is Cauchy in the space $\mathscr{L}(\lambda ; X, X)$ with respect to convergence in mean.

Then the function $f$ is the Fourier transform of a function belonging to the space $\mathscr{L}(\lambda ; X, Y)$.

Proof. The Cauchy net $\left\{\Phi_{\alpha}(f)\right\}_{\alpha \in \mathrm{A}}$ has a limit, $F$, in the complete space $\mathscr{L}(\lambda ; X, Y)$ (cf. Theorem 1.1). It follows from [10, Theorem 2(a)] that, for every $\theta \in \Theta$,

$$
\langle\theta, f(\gamma)\rangle=\int_{G}\langle\theta, F(g)\rangle(-g, \gamma) d g=\langle\theta, \hat{F}(\gamma)\rangle \quad(\gamma \in \Gamma)
$$

Since $\Theta$ is a total subset of $X^{\prime}$, we have $f=\hat{F}$.

Theorems 2.1 and 2.2 can be used to characterize those $X$-valued functions on $\Gamma$ which are Fourier transforms of $X$-valued Bochner $\lambda$-integrable functions on $G$. This gives a slight extension of the characterization in [1], where it was assumed that $G$ is a metrizable locally compact Abelian group.

Corollary 2.3. A function $f: \Gamma \rightarrow X$ is the Fourier transform of an $X$-valued, Bochner 
$\lambda$-integrable function on $G$ if and only if the following conditions hold:

(i) the function $f$ is bounded and continuous;

(ii) for every $\alpha \in A$, the function $\Phi_{\alpha}(f): G \rightarrow X$, given by (1), is Bochner $\lambda$-integrable, and the net $\left\{\Phi_{\alpha}(f)\right\}_{\alpha \in \mathrm{A}}$ is Cauchy with respect to the Bochner seminorm; that is,

$$
\lim _{\alpha, \beta \in A} \int_{G}\left|\Phi_{\alpha}(f)-\Phi_{\beta}(f)\right| d \lambda=0 .
$$

Proof. To prove the 'only if' part, take a Bochner $\lambda$-integrable function $F: G \rightarrow X$ such that $\hat{F}=f$. Statement (i) is a consequence of Theorem 2.1. Now, given $\varepsilon>0$, choose a Bochner $\lambda$-integrable, Borel simple function $H: G \rightarrow X$ for which

$$
\int_{G}|F-H| d \lambda<\varepsilon
$$

By the argument in the proof of Theorem 2.1,

$$
\int_{G}\left|\Phi_{\alpha}(f)-\Phi_{\alpha}(\hat{H})\right| d \lambda \leq \int_{G}|F-H| d \lambda,
$$

which implies statement (ii), since $H$ is a simple function.

To prove the 'if' part, take a Bochner $\lambda$-integrable function $F: G \rightarrow X$ such that

$$
\lim _{\alpha \in A} \int_{G}\left|F-\Phi_{\alpha}(f)\right| d \lambda=0 .
$$

It then follows from Theorem 2.2 that $f=\hat{F}$.

The author wishes to thank Professor Igor Kluvánek and Werner Ricker for valuable advice and many helpful discussions.

\section{REFERENCES}

1. Z. Bukovská, Characterization of Fourier transforms of vector valued functions and measures, Mat. Časopis Sloven, Akad. Vied., 20 (1970), 109-114.

2. H. Cramér, On the representations of a function by certain Fourier integrals, Trans. Amer. Math. Soc. 49 (1939), 191-201.

3. J. Diestel and J. J. Uhl, Jr., Vector measures, Math. Surveys, 15, (Amer. Math. Soc., Providence, 1977).

4. E. Hewitt and K. A. Ross, Abstract harmonic analysis II, Grundlehren, 152, (SpringerVerlag, 1970).

5. I. Kluvánek, Characterization of Fourier-Stieltjes transforms of vector and operator-valued measures, Czechoslovak Math. J., 17 (92) (1967), 261-277.

6. I. Kluvánek, Fourier transforms of vector-valued functions and measures, Studia Math., 37 (1970), 1-12.

7. S. Okada, Integration of vector-valued functions, in Measure theory and its applications, Lecture Notes in Mathematics No. 1033 (Springer-Verlag, 1983), 247-257.

8. B. J. Pettis, On integration in vector spaces, Trans. Amer. Math. Soc., 44 (1938), 277-304.

9. W. Rudin, Fourier analysis on groups, (Interscience, New York, 1960). 
10. A. B. Simon, Cesàro summability on groups: characterization and inversion of Fourier transforms, in Function algebra (Scott, Foresman and Company, Glenview, 1966), 208-215.

11. J. S. W. Wong, On a characterization of Fourier transforms, Monatsh. Math., 70 (1966), 74-80.

Department of Mathematical Sciences

San Diego State UnIVERSITY

San Diego, CA 92182-0314

U.S.A. 